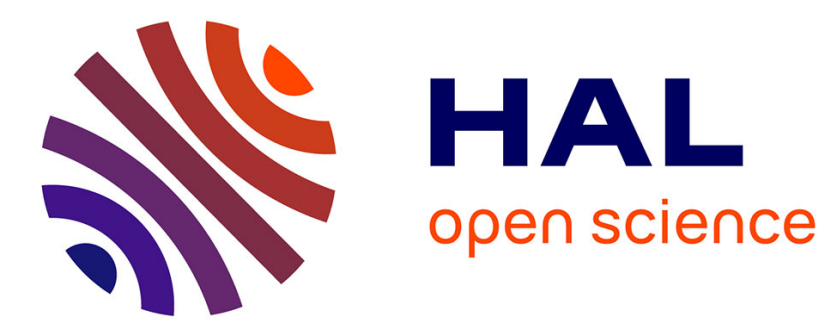

\title{
A Deep Point Cloud Geometry Coding Toolbox
}

\author{
Maurice Quach, Giuseppe Valenzise, Frédéric Dufaux
}

\section{To cite this version:}

Maurice Quach, Giuseppe Valenzise, Frédéric Dufaux. A Deep Point Cloud Geometry Coding Toolbox. IEEE International Conference on Multimedia \& Expo Workshops (ICMEW), Jul 2021, Shenzhen, China. pp.1-2, 10.1109/ICMEW53276.2021.9455986 . hal-03231402

\section{HAL Id: hal-03231402 \\ https://hal.science/hal-03231402}

Submitted on 20 May 2021

HAL is a multi-disciplinary open access archive for the deposit and dissemination of scientific research documents, whether they are published or not. The documents may come from teaching and research institutions in France or abroad, or from public or private research centers.
L'archive ouverte pluridisciplinaire HAL, est destinée au dépôt et à la diffusion de documents scientifiques de niveau recherche, publiés ou non, émanant des établissements d'enseignement et de recherche français ou étrangers, des laboratoires publics ou privés. 


\title{
A DEEP POINT CLOUD GEOMETRY CODING TOOLBOX
}

\author{
Maurice Quach Giuseppe Valenzise Frédéric Dufaux \\ Université Paris-Saclay, CNRS, CentraleSupélec, Laboratoire des signaux et systèmes, \\ 91190, Gif-sur-Yvette, France.
}

\begin{abstract}
This short paper describes a TensorFlow toolbox for point cloud geometry coding based on deep neural networks. This coding method employs a deep auto-encoder trained with a focal loss to learn good representations for voxel occupancy. The software provides several coding parameters to achieve different rate-distortion trade-offs, and comes with pre-trained models to reproduce the results of the published paper. It also offers a number of utility functions for evaluating and comparing the codec. To our knowledge, this is the first publicly available open-source toolbox for deeplearning-based point cloud coding.
\end{abstract}

\section{DESCRIPTION AND OVERVIEW}

Deep point cloud compression (PCC) is an emerging topic in multimedia coding, in particular for what concerns the coding of voxelized point clouds geometry $[1,2,3]$. As in other research fields, reproducing the results of existing approaches is of paramount importance to conceive new coding algorithms, especially when using deep neural networks, where the large number of variables involved makes it difficult to compare methods. This open source package offers a toolbox to compress point cloud geometry using deep neural networks. It implements all the contributions and experiments in our previous work [4]. The point cloud is partitioned into blocks and each block is compressed with a convolutional neural network, optimized with respect to a focal loss. In order to achieve different rate-distortion points, we train a network for each desired rate-distortion trade-off. At the decoder side, several parameters can be modified to adapt to the local point cloud density (balancing weight in the focal loss, optimal thresholding). Details on the choice of these parameters are reported in [4]. We complete the toolbox with a number of utility functions for evaluating and comparing the performance with other methods; we also provide pre-trained models and the datasets used to obtain them. We also provide instructions for users that want to retrain the networks on their own datasets.

We make the entire source code available at https:// github.com/mauriceqch/pcc_geo_cnn_v2 under the MIT

Funded by the ANR ReVeRy national fund (REVERY ANR-17-CE230020). ICME2021 "Point cloud capture and compression" track.

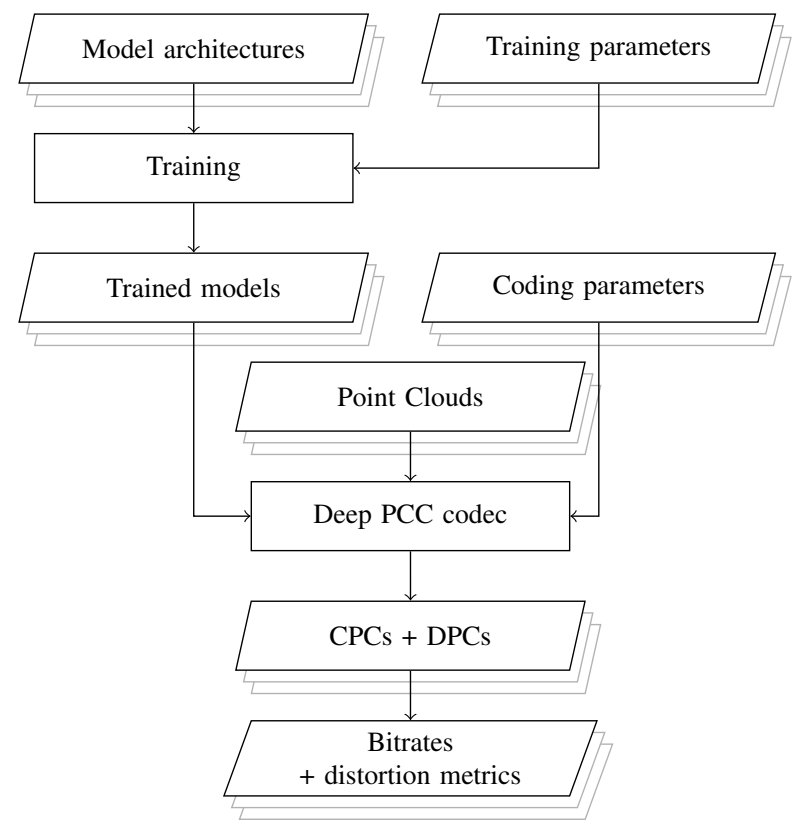

Fig. 1. System overview for the open source package. CPC stands for compressed PC and DPC for decompressed PC.

license. We also provide an overview of the package in Figure 1 . The source code contains a working implementation of all the compression models (conditions) described in the paper and includes the necessary code to reproduce all the experiments. Also, these experiments can easily be extended with other models or to other test point clouds by changing the configuration file ev_experiment.yml.

This open source package is intended for researchers and engineers working on PCC. It has two main purposes: i) facilitating the reproduction and comparison with the family of codecs in [4]; ii) providing the tools and a starting point to foster new research on deep PCC. In particular, the adopted licensing scheme allows the use of this software as a base for implementation in an industrial context.

\section{EXAMPLE OF UTILIZATION}

The package provides tools for each step of PCC. In particular, tools are provided to train the compression models, en- 


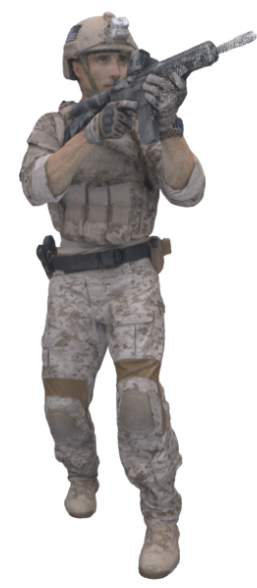

(a) Original

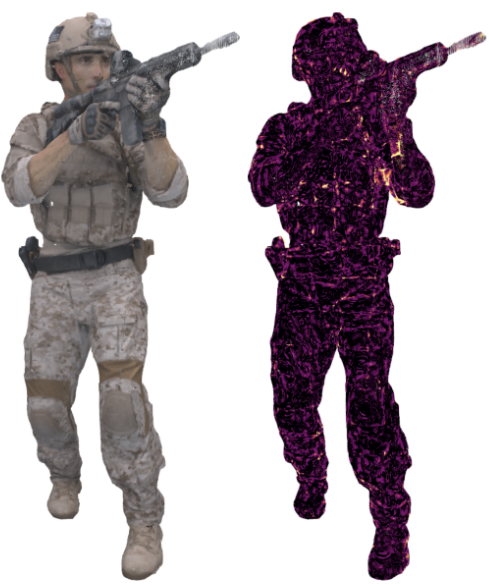

(b) c6 (D1 $69.59 \mathrm{~dB}, 0.194 \mathrm{bpp)}$
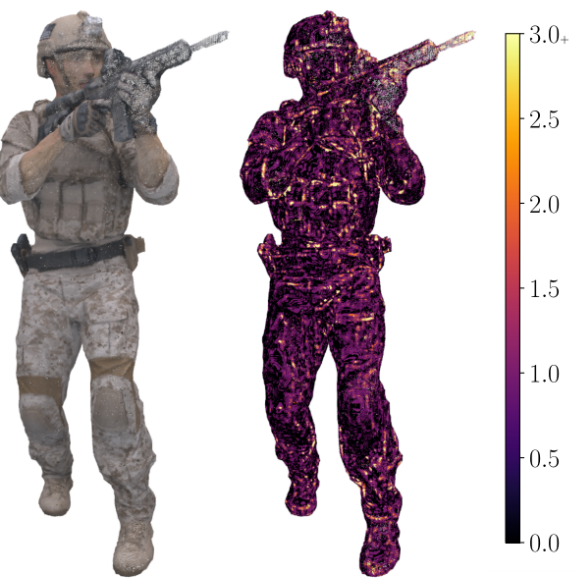

(c) G-PCC Trisoup (D1 $65.87 \mathrm{~dB}, 0.188 \mathrm{bpp})$

Fig. 2. Qualitative evaluation on "soldier_vox10_0690". For our method and G-PCC Trisoup (baseline), we show the decompressed point cloud and its D1 squared errors. The errors are displayed according to the color scale on the right and are truncated to the 99th percentile (3.0). In parentheses, we specify the D1 PSNR along with the number of bits per input point (bpp).

code and decode PCs and evaluate them. Note that the package works with voxelized point clouds.

\subsection{Training}

In order to train a compression model, we need a model architecture and training parameters. Model architectures are defined in model_configs.py and are identified by a unique name. The training parameters include the training dataset, the rate-distortion tradeoff parameter $(--1 \mathrm{mbda})$, the focal loss parameters (--alpha and --gamma), the batch size and the model architecture (--model_config). Usually, multiple compression models are trained for a single architecture with different rate-distortion trade-offs. Below is a sample training command:

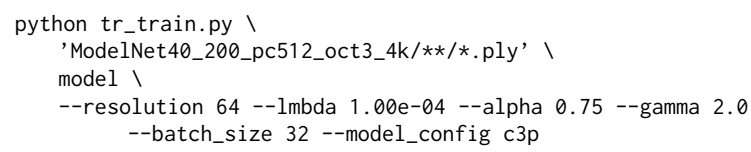

\subsection{Coding a point cloud}

Then, we can compress and decompress a point cloud using the previously trained model. Coding parameters include the model architecture, the octree level used for block partitioning and the optimal thresholding target metrics (--opt_metrics). In the following example, the software encodes and decodes a PC, employing the point-to-point metric (D1) to optimize the thresholding at the decoder:

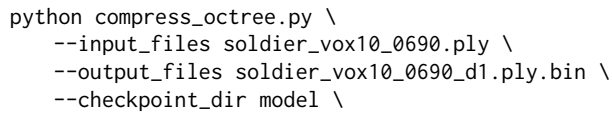

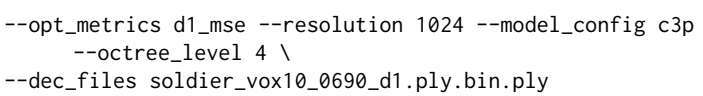

\subsection{Visualization and evaluation}

For visualization, we can transfer the colors from the original to the decompressed point cloud with the following command:

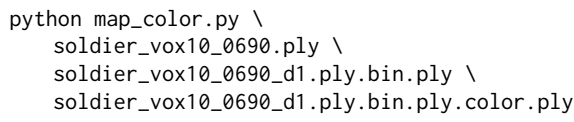

Then, we can use the mpeg-pcc-dmetric to compute distortion metrics:

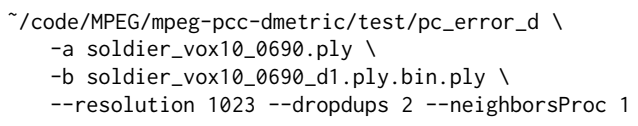

A sample compressed point cloud is shown in Figure 2; it is compared with the original and the same point cloud compressed with G-PCC. More details are available on Github.

\section{REFERENCES}

[1] M. Quach, G. Valenzise, and F. Dufaux, "Learning Convolutional Transforms for Lossy Point Cloud Geometry Compression," in 2019 IEEE Intl. Conf. on Image Process. (ICIP), Sept. 2019, pp. 4320-4324, ISSN: 1522-4880.

[2] A. F. R. Guarda, N. M. M. Rodrigues, and F. Pereira, "Point Cloud Coding: Adopting a Deep Learning-based Approach," in 2019 Picture Coding Symposium (PCS), Nov. 2019, pp. 1-5, ISSN: 2330-7935.

[3] J. Wang et al., "Learned Point Cloud Geometry Compression," arXiv:1909.12037 [cs, eess], Sept. 2019.

[4] M. Quach, G. Valenzise, and F. Dufaux, "Improved Deep Point Cloud Geometry Compression," in 2020 IEEE 22nd Intl. Workshop on Multimedia Signal Process. (MMSP), Sept. 2020, pp. 1-6, ISSN: 2473-3628. 